\title{
KINETICS OF PARTICLE CHARGING IN AN ELECTROSTATIC FIELD OF SEPARATORS
}

\author{
ANATOLY I. MESENYASHIN* \\ Mekhanobr-Tekhnika Co, 3, 28 Liniya, St. Petersburg, 199106, Russia
}

(Received 6 December 2001; Accepted 1 January 2002)

\begin{abstract}
With reference to electrostatic separation, expressions are considered for computation of kinetics of particle charging in electrostatic field and corona discharge field. Particles are represented as three-axis ellipsoids, which permits to approximate the particles of various shapes. Volume conductance and dielectric permittivity, of both particles and ambient medium are taken into consideration. Total charges and free charges of particles with high and low conductance are considered. General expression permitting to define particle charge in a time-varying electric field has been derived. An example of computation is considered, when external field varies sinusoidally, and dependence of the particle charges on the external field duration and variation rate is shown.
\end{abstract}

Keywords: Electrostatic separation; Dielectric permittivity; Conductance; Charging of particles

\section{INTRODUCTION}

A significant difference in magnitude of charges of separated particles is a necessary requirement for electrostatic separation. Moreover, separation is most efficient if the separated particles have unlike charges. The possibility of separation depends on the difference in maximum charges and in the rates of both charging and discharging of particles. Therefore it is important to estimate such values, as they permit choice of both electrostatic separator design and electrostatic separation parameters.

\footnotetext{
*E-mail: anatmes@robotek.ru
} 
Polarity and magnitude of particle charges depend on the values of conductance and dielectric permittivity of both the particles and the ambient medium. Particle shape, external field non-uniformity and the rate of external field amplitude variation exert a significant influence on the particle charge. The operating modes of electrostatic separation may employ different values of charge both with steady state and transient parameters of the electrostatic field.

However, in theoretical physics, particles are usually considered as perfect conductors or non-conductors charged in a non-conducting medium by a constant external field [1,2]. The same approach was widespread in the analysis of the particle behaviour in electrostatic separation for a long time [3-5]. This did not permit special features of particle behaviour in an electric field to be revealed and often led to incorrect determination not only of magnitude, but also of the sign of the particle charge.

This article examines the kinematics of particle charging and discharging, taking into account the volume conductance and dielectric permittivity both of particles of various shapes and of the ambient medium with consideration of the time variation of external field.

\section{DEVELOPMENT OF THEORY OF PARTICLES CHARGING IN ELECTROSTATIC SEPARATORS}

Concepts of total charge and free charge are used. The total charge consists of the free charge and the polarization charge. The total charge of a particle determines the magnitude of the force between the particle and the electrode [6-8], as well as that of electric force depending on the nonuniformity of the electric field, i.e., electric intensity gradient.

At a distance from the electrodes, the summarized contribution of the polarization charges to the external force is equal to zero, since unlike polarization charges are concentrated on opposite points of the particle.

The maximum quantity of a charge of high-conductivity sphere of radius $b_{1}$ positioned on an electrode in a uniform field $E_{k}$ is estimated by the following equation [9]

$$
q_{t \infty}=-2 \pi^{3} \varepsilon_{0} b_{1} E_{k} / 3 .
$$


Since the 1970s formulae that take into consideration the volume conductance of a particle and medium, as well as particle shape [6-8,10], have gained ground.

Particles are usually represented as spheres or ellipsoids. Representation of particles as ellipsoids permits approximation for particles of different shapes by varying the ratio of the lengths of axes.

Let us consider the charging of a particle shaped as a semi-ellipsoid and positioned on a grounded electrode (Fig. 1). Let $a, b$ and $c$ denote semi-ellipsoid axes. Axis $a$ is oriented along the external uniform field $E_{k}$.

If the constant external field is energized at moment $t=0$, the total charge variation in time will be described by expression $[6,8]$ :

$$
q_{t}(t)=q_{t \infty}+\left(q_{t 0}-q_{t \infty}\right) e^{-t / \tau}
$$

where $q_{t \infty}, q_{t 0}$ are the maximum and initial charges and $\tau$ is the time constant of the assumed process.

If the particle has not been previously charged, the initial (at $t=0$ ) total charge of a particle depends on the dielectric permittivity of a particle $\varepsilon_{1}$ and that of the medium $\varepsilon_{2}$ :

$$
q_{t 0}=n_{2} E_{k} / n_{1},
$$

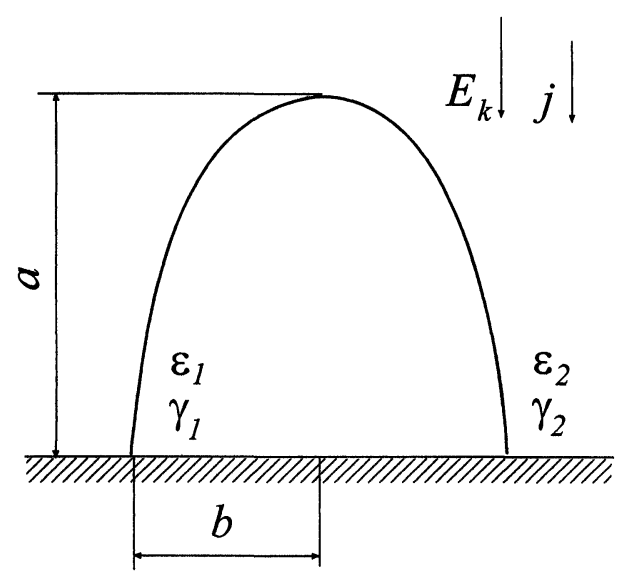

FIGURE 1 A particle on a collecting electrode. 
where $n_{1}=\varepsilon_{1} d_{a}+\varepsilon_{2}\left(1-d_{a}\right) ; n_{2}=\varepsilon_{0} \pi b c\left(\varepsilon_{2}-\varepsilon_{1}\right)$; with $d_{a}$ being the depolarization factor depending on the ratio of the semi-ellipsoid axes [1].

The maximum total particle charge (at $t=\infty$ ) depends on the conductance values

$$
q_{t \infty}=m_{2} E_{k} / m_{1},
$$

where $m=\gamma_{1} d_{a}+\gamma_{2}\left(1-d_{a}\right) ; m_{2}=\varepsilon_{0} \pi b c\left(\gamma_{2}-\gamma_{1}\right)$.

The charging and discharging time constant is equal to

$$
\tau=n_{1} / m_{1} \text {. }
$$

As for the free charge, if a particle has not been charged in the initial period, the kinematics of charging will be described by this equation:

$$
q_{f}(t)=q_{f \infty}\left(1-e^{-t / \tau}\right),
$$

where $\tau$ is determined by Eq. (5), and the magnitude of the maximum free charge $q_{f \infty}$ in a constant external field $E_{k}=$ const is equal to

$$
q_{f \infty}=\left(\varepsilon_{1} \gamma_{2}-\varepsilon_{2} \gamma_{1}\right) \varepsilon_{0} \pi b c E_{k} / m_{1} .
$$

Similar tasks are dealt with in the theory of electrophoresis, but only regarding spherical particles [11].

A recently published article [12] shows that electric forces acting on particles in a non-uniform field depend on the history of the particles' motion, i.e., not only on the initial charge, but also on the rate of motion of the particles. In particular, particle oscillations may take place in a non-uniform electrostatic field under certain conditions.

It is noteworthy that the needs of electrostatic separation have led to the development of theories that are of interest in other fields, e.g., it is possible to suppose that a phenomenon of particle oscillations in an electrostatic field will be used in engineering, although to a smaller extent than mechanical or electromagnetic oscillations.

Another interesting example is that studies of the charging of composite particles, in particular shells [13], permitted the development of a modern physical model of ball lightning [14] and also explanation of the nature of certain unidentified flying objects. This is explained by the fact that electrostatic separation involves naturally created substances, and not ideal ones. 


\section{THE CHARGING OF PARTICLES IN A SINUSOIDALLY VARYING FIELD}

In the processing practice, the external electric field usually varies with time. For example, during the transport of particles in a separator from a feeder to a separation zone the electric field varies from 0 to a maximum value, and then falls off.

The total charge may be determined by this differential equation:

$$
n_{1} d q_{t} / d t+m_{1} q_{t}=n_{2} d E_{k} / d t+m_{2} E_{k}
$$

Solution of this equation applying Laplace transformation will give

$$
q_{t}=\frac{n_{2}}{n_{1}} E_{k}(t)+\left[n_{1} q_{t o}-n_{2} E_{k}(0)\right] \frac{1}{n_{1}} e^{-\left(m_{1} / n_{1}\right) t}+\frac{m_{2} n_{1}-m_{1} n_{2}}{n_{1}^{2}} e^{-\left(m_{1} / n_{1}\right) t} I(t),
$$

where

$$
I(t)=\int_{0}^{t} e^{m_{1} \theta / n_{1}} E_{k}(\theta) d \theta
$$

Free charge $q_{f}$ is determined taking into account that its dependence on the total charge $q_{t}$ assumes the form

$$
q_{f}=-\left(n_{2} / \varepsilon_{0}\right) E_{k}(t)+n_{1} q_{t} / \varepsilon_{0} .
$$

Formula (9) holds true for the arbitrary function $E_{k}(t)$. An expression for $q_{t}(t)$ may be obtained in elementary functions when the external electric field varies sinusoidally.

Let us consider special cases of determination of the function $I(t)$.

The duration of the external field is equal to the half-cycle $(T)$ of a sinusoid with amplitude $E_{m}$. Namely, if

$$
E_{k}(t)=\left\{\begin{array}{cc}
E_{m} \sin \left(\frac{t}{T} \pi\right) & 0 \leq t<T, \\
0 & t>T
\end{array}\right.
$$


then for cycle $0 \leq t<T$

$$
I(t)=\frac{n_{1} T E_{m}}{n_{1}^{2} \pi^{2}+m_{1}^{2} T^{2}}\left[n_{1} \pi-n_{1} \pi e^{\mu} \cos \left(\frac{\pi t}{T}\right)+m_{1} T e^{\mu} \sin \left(\frac{\pi t}{T}\right)\right],
$$

where $\mu=m_{1} T / n_{1}$,

for $t \geq T$

$$
I(t)=n_{1}^{2} \pi T E_{m}\left(1+e^{\mu}\right) /\left(n_{1}^{2} \pi^{2}+m_{1}^{2} T^{2}\right) .
$$

If the external field increases sinusoidally to a maximum magnitude and after that remains constant, we obtain

$$
E_{k}(t)=\left\{\begin{array}{cc}
E_{m} \sin \left(\frac{t}{T} \pi\right) & 0 \leq t<T / 2 \\
E_{m} & T / 2 \leq t
\end{array}\right.
$$

Under these conditions, in a time period $0 \leq t<T / 2$ the expression for $I(t)$ will have the same value, as in the previous case (14). And for $T / 2 \leq t$ we obtain

$$
I(t)=\left[\frac{n_{1}^{2} \pi T-n_{1} m_{1} T^{2} e^{\mu / 2}}{n_{1}^{2} \pi^{2}+m_{1}^{2} T^{2}}+\frac{n_{1}}{m_{1}}\left(e^{\mu}-e^{\mu / 2}\right)\right] E_{m} .
$$

For an external field which is energized step-wise, i.e., the rate of increase $E_{k}(t)=E_{m}$ with $t \geq 0$, we obtain

$$
I(t)=\left(E_{m} n_{1} / m_{1}\right)\left(e^{\mu}-1\right) .
$$

The magnitude of chargers regarding this case has been considered above.

\section{RESULTS AND DISCUSSION}

The quoted formulae may be used for analysis of particle charging in certain electric fields. 


\subsection{Maximum Magnitudes of Particle Charges}

Let us consider the maximum $(t \rightarrow \infty)$ magnitudes of charges with $E_{k}=$ const. According to Eq. (4), for the case when the medium conductance $\left(\gamma_{2}\right)$ may be ignored, the magnitude of the maximum total charge will be equal to

$$
q_{t \infty}=-\varepsilon_{0} \pi b c E_{k} / d_{a}
$$

The magnitude of the maximum charge of such particles depends on their shape, size and $E_{k}$ the particle charge sign coinciding with that of the collecting electrode, upon which particles are located.

According to Eq. (7), the magnitude of the maximum free charge in a non-conducting medium is equal to

$$
q_{f \infty}=-\varepsilon_{0} \varepsilon_{2} \pi b c E_{k} / d_{a}
$$

and in air $\left(\varepsilon_{2}=1\right)$ it is of the same magnitude as the maximum total charge.

Consequently, with $\gamma_{2}=0$, particles of the same shape and size, but of different conductance, will acquire equal charges upon completion of charging.

Let us compare the maximum charge of a half-sphere with the ratio axis of $a=2 b=2 c$ with the charge of the sphere computed using Eq. (1) [9]. The difference in charges computed by (1) and (18) is only $12 \%$, indicating, that the half-sphere provides a close approximation of the spherical particle.

\subsection{Particle Charging in a Corona Discharge Field and the Effect of Medium}

In the practice of electrostatic separation, the particle charging often takes place in a corona discharge field in air, although installations featuring particle charging in medium of other gases have been described [15]. Commercial-scale drum corona separators with the medium of inert gases (argon and helium) exist. In dielectric separation, particles are separated in non-conducting liquids with specially chosen values of $\varepsilon_{2}$. 
As may be seen from the Eq. (7), the sign of the free charge depends on the sign of $\varepsilon_{1} \gamma_{2}-\varepsilon_{2} \gamma_{1}$.

In the corona discharge field the medium conductance $\gamma_{2}$ depends on the corona discharge current density $j$ and the external field intensity $E_{\kappa}$ :

$$
\gamma_{2}=j / E_{\kappa}
$$

In electrostatic separation in air typical values of $j$ and $E_{\kappa}$ are equal to $10^{-4} \mathrm{~A} / \mathrm{m}$, and $4 \times 10^{-5} \mathrm{~V} / \mathrm{m}$, respectively.

With $\varepsilon_{2} \gamma_{1}>\varepsilon_{1} \gamma_{2}$, the particle will acquire charge of the same sign as that of the collecting electrode and will break loose from that electrode. This is typical for conducting particles. With $\varepsilon_{2} \gamma_{1}<\varepsilon_{1} \gamma_{2}$ the particle will acquire unlike charge as regards the collecting electrode and will be attracted to that electrode. This is typical for non-conducting particles. By varying the magnitude of corona discharge current, it is possible to change both the magnitude and sign of the particle charge. This circumstance determines the stability of the separation process of conductors in drum corona separators. The corona discharge current depends on the design of the corona-forming electrode, the voltage applied to the corona-forming electrode, as well as the composition of the gaseous medium.

The value of $\gamma_{2}$ may be increased by means of inert gases. For example, in argon and helium the corona discharge current is 10 to 20 times higher than in air. In addition, the voltage is 2 to 3 times lower than in air. This permits separation of metal powders from oxides.

\subsection{Kinetics of Particle Charging in a Constant Field}

The maximum charge $q_{f \infty}$ is acquired in a certain time interval. Thus during a time interval $\tau$ the particles will be charged to $0.368 q_{f 0}$, and $0.99 q_{f \infty}$ charge will be acquired in $4.6 \tau$.

This is illustrated in Figs. 2 and 3, which show the charging of the conducting and non-conducting particles of size $a=2 b=2 c=0.5 \times$ $10^{-3} \mathrm{~m},\left(d_{a}=0.172\right)$, with $E_{\kappa}=4 \times 10^{5} \mathrm{~V} / \mathrm{m}$. In an electrostatic field the parameters of the medium (air) are assumed to be: $\varepsilon_{2}=1, \gamma_{2}=0$, and in the corona discharge field: $\varepsilon_{2}=1, \quad \gamma_{2}=2.5 \times$ 
Time $t, s$

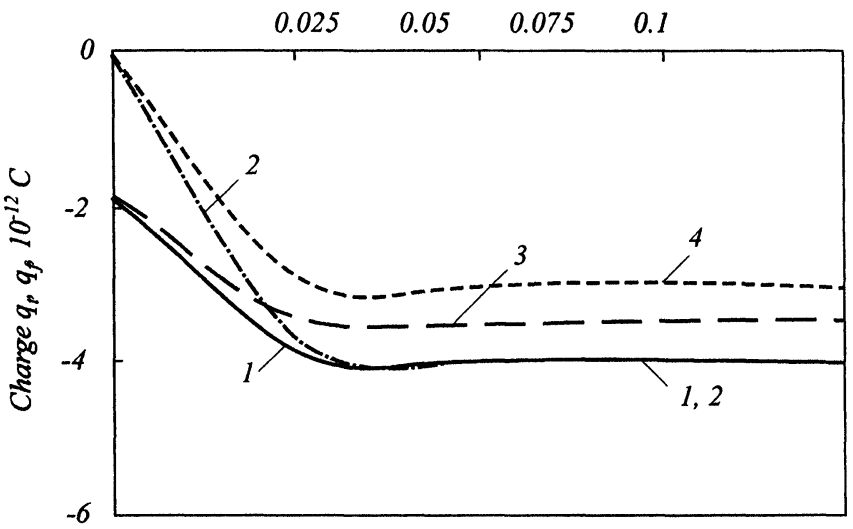

FIGURE 2 Kinetics of particle charging in a constant field. 1 - Total charge $q_{t}$ in electrostatic field. 2 - Free charge $q_{f}$ in electrostatic field. 3 - Total charge $q_{t}$ in corona discharge field. 4 - Free charge $q_{f}$ in corona discharge field.

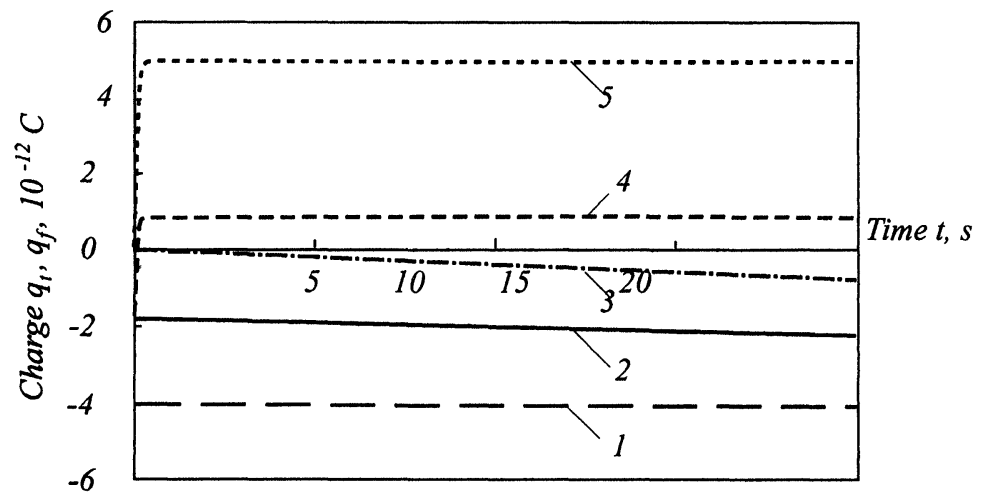

FIGURE 3 Kinetics of charging of non-conducting particles in a constant field. 1 Maximum total charge $q_{t \infty}$ and maximum free charge $q_{f \infty}$ in electrostatic field. 2 - Total charge $q_{t}$ in electrostatic field. 3 - Free charge $q_{f}$ in electrostatic field. 4 - Total charge $q_{t}$ in corona discharge field. 5 - Free charge $q_{f}$ in corona discharge field.

$10^{-10} \mathrm{Ohm}^{-1} \mathrm{~m}^{-1}$. In computations a conducting particle is assumed to have $\varepsilon_{1}=6, \gamma_{1}=10^{-8} \mathrm{Ohm}^{-1} \mathrm{~m}^{-1}$, and non-conducting one: $\varepsilon_{1}=6$, $\gamma_{1}=10^{-12} \mathrm{Ohm}^{-1} \mathrm{~m}^{-1}$.

At an initial period of time $(t=0)$ of the magnitude, total charge $q_{t 0}$ depends on dielectric permittivity values (3) and is not equal to zero 
(Fig. 2, curves 1,3; Fig. 3, curves 2,4). The magnitude free charge $q_{f 0}$ with $t=0$ is equal to zero, if the particle has not been previously charged (Fig. 2, curves 2,4; Fig. 3, curves, 3,5).

Regarding the above parameters, conducting particles (Fig. 2) in an electrostatic field and in a corona discharge field are characterized by similar charging time constants $(\tau)$, namely, upon constant voltage energization, $\tau=0.0096 \mathrm{~s}$ and $\tau=0.0085 \mathrm{~s}$, respectively.

Non-conducting particles (Fig. 3) in an electrostatic field and in a corona discharge field acquire unlike charges on a collecting electrode. Moreover, the magnitude of the free charge in the corona discharge field (Fig. 3, curve 5) may exceed the maximum value of the charge in an electrostatic field (Fig. 3, curve 1). Non-conducting particles in these fields are characterized by essentially different charging time constants, namely in an electrostatic field $\tau=95.7 \mathrm{~s}$ (Fig. 3, curves 2, 3), and in a corona discharge field $\tau=0.079$ (Fig. 3, curves 4, 5). As regards non-conducting particles, their time of charging in an electrostatic field depends on the particle conductance and their time of charging in a corona discharge field on the medium conductance.

In an electrostatic field $\left(\gamma_{2}=0\right)$, different rates of charging are used for conductors and non-conductors to charge particles of the same shape with unlike charges on the collecting electrode. In the corona discharge field, unlike charges are obtained by charging particles to maximum charges.

\subsection{Kinetic of Particle Charging in an Alternating Field}

During electrostatic separation the particle retention time in electric field is 0.01 to $1 \mathrm{~s}$. The smaller value of this parameter is characteristic for constructions featuring particles in contact with the collecting electrode while the higher value is characteristic of designs where particles move without contact with the collecting electrode. Analysis of particle charging in a sinusoidal field permits examination of the effects of both charging time and variation rate of external field intensity on particle charges.

If conducting particles having the above parameters are placed in an external electrostatic field $E_{\kappa}=E_{m} \sin (\pi t / T)$ with $T=0.1 \mathrm{~s}$, the particle charges increase from zero to the maximum magnitude 
Time $t, s$

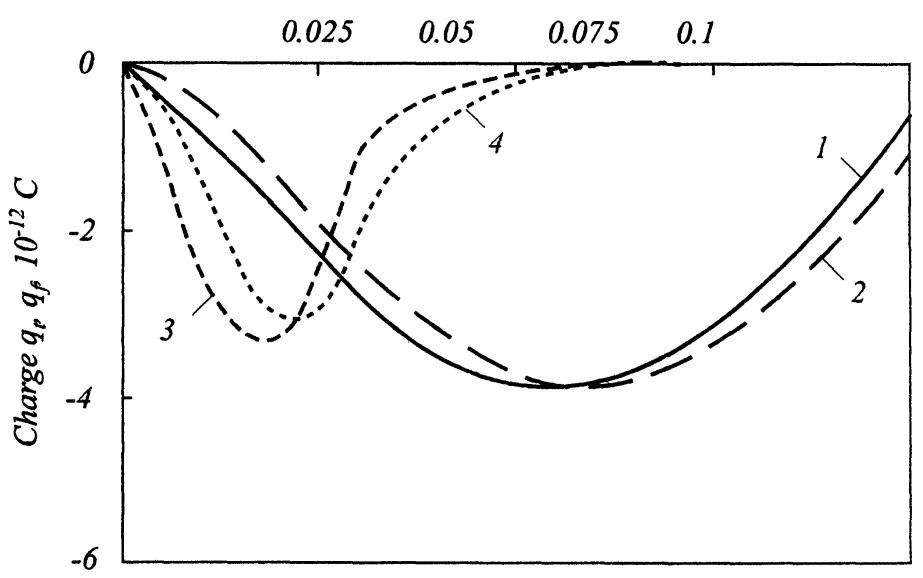

FIGURE 4 Kinetics of charging conducting particles in a sinusoidally varying electrostatic field. 1 - Total charge $q_{t}$ with $T=0.1 \mathrm{~s}$. 2 - Free charge $q_{f}$ with $T=0.1 \mathrm{~s} .3-$ Total charge $q_{t}$ with $T=0.03 \mathrm{~s}$. $4-$ Free charge $q_{f}$ with $T=0.03 \mathrm{~s}$.

(Fig. 4, curves 1,2). This maximum magnitude is practically equal to the maximum charge acquired by a particle in an external constant field (Fig. 2, curves 1,2). In an external electric field with $T=0.03 \mathrm{~s}$ (Fig. 4, curves 3,4 ) the maximum magnitude of the charge will be smaller than that in a constant field. Both a gradual increase of the external field and the short-time action of the field upon particles may prevent them from acquiring maximum charges during charging.

After the external field $E_{\kappa}$ decreases to zero, a particle may retain the free charge. Thus, with $t=T=0.1 \mathrm{~s}$ (Fig. 4, curve 2) the value of the free charge will amount to $27 \%$ of the maximum charge value acquired in a constant field. And with $t=T=0.03 \mathrm{~s}$ (Fig. 4, curve 4) the value of free charge will amount to $52 \%$ of such a maximum charge.

As regards various constructions, e.g., drum separators, it is expedient to execute either rapid or gradual increase of the electric field, depending on the feed material and the required results of separation. Thus, if conducting and non-conducting particles of the same shape, size and dielectric permittivity $\left(\varepsilon_{1}\right)$ are placed in a constant field, they will acquire equal total charges in the initial period (Fig. 2, curve 1; Fig. 3, curves 2, 4). Particles characterized by high values of $\varepsilon_{1}$ and oblong shape, orientated along the field, will acquire a large 
charge (Eq. (3)) and may break off from the drum. It is precisely total charges that determine electric forces acting on particles in contact with an electrode [7]. Differences in magnitude of the total charges of conducting and non-conducting particles of the same shape and dielectric permittivity become apparent only with time (Eq. (2)). However, in an electrostatic field the maximum charges of such particles are determined by the expression (18).

Let us consider methods for comparison of magnitudes of charges with instantaneous increase (Eq. (17)) and sinusoidal increase Eqs. (15-16) of the external field.

In both cases magnitudes $E_{\kappa}(0)=0$, and with $t>T / 2$ values $E_{\kappa}(t)$ are equal. According to (8), the difference in the value of charges at the same moments of time will depend on the value of the $I(t)$. An expression for the difference in charges has the following form:

$$
\delta q=\frac{m_{2} n_{1}-m_{1} n_{2}}{n_{1} m_{1}} E_{m} e^{m_{1} t / n_{1}}\left[e^{\mu / 2}-1-\frac{\pi \mu-\mu^{2} e^{\mu / 2}}{\pi^{2}+\mu^{2}}\right] .
$$

An immediate construction of a graph of the function in square brackets shows that it is positive for any $\mu$. Consequently, the sign of value $\delta q(21)$ is determined, for any $T$, by the sign of expression

$$
\begin{aligned}
\chi & =m_{2} n_{1}-m_{1} n_{2} \\
& =\varepsilon_{0} \pi b c\left[\varepsilon_{2}\left(\gamma_{1}-\gamma_{2}\right)+\gamma_{2}\left(\varepsilon_{1}-\varepsilon_{2}\right)+2 d_{a}\left(\varepsilon_{1}-\varepsilon_{2}\right)\left(\gamma_{1}-\gamma_{2}\right)\right] .
\end{aligned}
$$

With reference to one particle, if $\chi>0$, the charge in the former case (instantaneous energization) is greater than in the latter (gradual energization). If $\chi>0$, the particle charge in the former case is smaller than in latter, with charging taking place in air medium. The latter case is possible only if the particle conductance is smaller, than that of air, bearing in mind a charge with the same value of $t$. Naturally, within limit $t \rightarrow \infty$ the difference between charges in two cases in question converges to zero owing to factor $e^{-m_{1} t / n_{1}}$.

Expression (22) permits consideration of the difference in charges of two particles with different properties at instantaneous and gradual energization of the field. If the signs of $\chi$ of the particles are negative, gradual energization with the greatest possible $T$ is preferable. This is of interest regarding particles with poor conductance. If the signs of $\chi$ are 
different, instantaneous energization is preferable for particle separation. In contrast to gradual energization, the particle charges that are already greater increase. Consequently, the difference in the charges of the particles is enhanced.

The electrostatic separation process is affected by numerous factors. The above examples illustrate the significance of electric field geometry and time of charging.

The charge of particles is the main physical parameter forming the basis of electrostatic separation. Nevertheless, such charges usually vary in the separation process. This feature distinguishes electrostatic separation from other main beneficiation processes, e.g., magnetic and gravity separation, since the principal parameters that form the basis for the latter processes are constant and time-independent.

\section{References}

[1] L.D. Landau and Ye.M. Livshits, Electrodynamics of Continuums, Pergamon Press, Oxford, 1984.

[2] J.A. Stratton, Electromagnetic Theory, Ch. I, III. McGraw-Hill Book Company, N.Y. - London, 1941.

[3] F. Fraas, Electrostatic Separation of Granular Materials, US Bureau of mines, Bul. 603, Ch. 3, 1962.

[4] C.Y. Cheng, Y.Z. Lin and W.B. Hu, A novel electrostatic separator and its application to benefication of fine minerals. Proc XVII Int. Min. Proc. Cong. Dresden, Vol. III, pp. 301-312, 1991.

[5] E.G. Kelly and D.J. Spottiswood, The theory of electrostatic separation: a review. Min. Eng., 2 (1989), 1-3.

[6] I.P. Versechagin, V.I. Levitov, G.Z. Mirzabekyan and M.M. Pashin, Fundamentals of Electro-Gas-Dynamics of Dispersions, (1974), Energiya, Moscow (In Russian).

[7] A.I. Mesenyashin, Electrostatic Separation in Strong Fields, (1978), Nedra, Moscow (In Russian).

[8] A.I. Mesenyashin, Charges of particles and electric forces in drum corona electric separator. Obog. Rud, 6 (1980), 33-37 (In Russian).

[9] N.N. Lebedev and I.P. Skalskaya, Force acting on conducting ball placed in plane capacitor field. J. Tech. Phys., 32 (1962), 375-378 (In Russian).

[10] T.B. Jones, Electromechanics of Particles. Cambridge University Press, New York, (1995).

[11] L. Benguigui and I.J. Lin, More about the dielectrophoretic force. J. Appl. Phys., 53 (1982), 1141-1143.

[12] A.I. Mesenyashin and E.B. Kremer, Motion of a sphere in non-uniform electrostatic field. Journal of Physics D., 33 (2000), 216-220.

[13] A.I. Mesenyashin, Dynamics of electric separation of composite particles in contact with electrode. Phys. \& Eng. Probl. Min. Min., 6 (1985), 87-92 (In Russian).

[14] A.I. Mesenyashin, Spherical formations in the atmosphere as a physical phenomenon. J. Electro., 36 (1995), 139-150.

[15] I.I. Inculet, Z. Kucerovski and M. Suzuki. Electric charging of particles near the corona glow region in air- $\mathrm{CO}_{2}$ mixtures. IEEE Trans. Ind. Appl., IA-15, 276-287. 


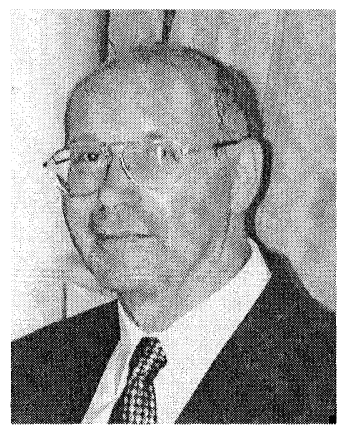

Anatoly I. Mesenyashin graduated from the Leningrad Polytechnical Institute. His main field of expertise and interest is the theory and practice of the application of high voltage techniques in materials processing. Dr. Mesenyashin is the author of fundamental research studies into physical electrostatics and its occurrence in some natural phenomena involving electricity. He played an important role in the development of the electrostatic separation technology in Russia. He has many years of experience in the design and engineering of electrostatic separators used for beneficiation of rare and non-ferrous metal ores, industrial minerals and wastes. $\mathrm{He}$ is the inventor of new high-capacity separators applicable to the recovery of high purity metallic and non-metallic powders. Dr. Mesenyashin is the author of the textbook Electrostatic Separation in High-Intensity Fields, which was published in 1977. $\mathrm{He}$ has more than 60 inventions to his credit and is the author of about 100 publications in scientific and technical journals. Dr. Mesenyashin is presently the Head of the Electrostatic Separation Laboratory in Mekhanobr-Tekhnika Co. 